\section{CD123 expression patterns and selective targeting with a CD123-targeted antibody-drug conjugate (IMGN632) in acute lymphoblastic leukemia}

Evgeniya Angelova, ${ }^{1}$ Charlene Audette ${ }^{2}$, Yelena Kovtun, ${ }^{2}$ Naval Daver, ${ }^{3}$ Sa A. Wang, ${ }^{1}$ Sherry Pierce, ${ }^{3}$ Sergej N. Konoplev, ${ }^{1}$ Haitham Khogeer, ${ }^{1}$ Jeffrey L. Jorgensen, ${ }^{1}$ Marina Konopleva, ${ }^{3}$ Patrick A. Zweidler-McKay, ${ }^{2}$ L. Jeffrey Medeiros, ${ }^{1}$ Hagop M. Kantarjian, ${ }^{3}$ Elias J. Jabbour ${ }^{3}$ and Joseph D. Khoury ${ }^{1}$

${ }^{1}$ Department of Hematopathology, The University of Texas MD Anderson Cancer Center, Houston, TX; 'ImmunoGen, Inc., Waltham, MA and ${ }^{3}$ Department of Leukemia, The University of Texas MD Anderson Cancer Center, Houston, TX, USA

\section{ABSTRACT}

he potential of CD123-targeted therapies in acute lymphoblastic leukemia/lymphoma remains largely unexplored. We examined CD123 expression levels in a large cohort of patients with acute lymphoblastic leukemia/lymphoma and assessed the in vitro impact of IMGN632, a conjugate of CD123-binding antibody with a novel DNAalkylating payload. CD123 expression on leukemic blasts was surveyed using multicolor/multiparameter flow cytometry. The in vitro effect of IMGN632 was evaluated on B acute lymphoblastic leukemia/lymphoma cell lines and primary B acute lymphoblastic leukemia/lymphoma blasts. The study cohort $(n=213)$ included 183 patients with B acute lymphoblastic leukemia/lymphoma and 30 with $\mathrm{T}$ acute lymphoblastic leukemia/lymphoma. CD123 expression was more prevalent in B acute lymphoblastic leukemia/lymphoma than in $\mathrm{T}$ acute lymphoblastic leukemia/lymphoma (164/183, $89.6 \%$ versus $13 / 30,43.3 \% ; P<0.0001)$, and within B acute lymphoblastic leukemia/lymphoma CD123 expression was more prevalent in Philadelphia chromosome-positive patients than in Philadelphia chromosome-negative patients ( $96.6 \%$ versus $86.3 \% ; P=0.033)$. In $\mathrm{T}$ acute lymphoblastic leukemia/lymphoma, 12/13 (92.3\%) patients with CD123-positive blasts had either early $\mathrm{T}$ precursor (ETP) or early nonETP immunophenotype. IMGN632 was highly cytotoxic to B acute lymphoblastic leukemia/lymphoma cell lines, with half maximal inhibitory concentrations $\left(\mathrm{IC}_{50}\right)$ between 0.6 and $20 \mathrm{pM}$. In five of eight patients' samples, low pico-molar concentrations of IMGN632 eliminated more than $90 \%$ of the B acute lymphoblastic leukemia/lymphoma blast population, sparing normal lymphocytes. In conclusion, CD123 expression is prevalent across acute lymphoblastic leukemia/lymphoma subtypes, and the CD123-targeted antibody-drug conjugate IMGN632 demonstrates promising selective activity in preclinical models of B acute lymphoblastic leukemia/lymphoma.

\section{Introduction}

Acute lymphoblastic leukemia (ALL) is an aggressive hematologic neoplasm arising from immature lymphoid precursors. In adults, $75 \%$ of ALL cases develop from B-cell precursors (B-ALL) and the remainder from T-cell precursors (TALL). B-ALL has been long known for its genetic heterogeneity and includes a subset of cases that harbors the $B C R-A B L 1$ fusion located on the derivative chromosome 22 (Philadelphia chromosome) resulting from $\mathrm{t}(9 ; 22)(\mathrm{q} 34.1 ; \mathrm{q} 11.2)$. Philadelphia chromosome $(\mathrm{Ph})$-positive $\left(\mathrm{Ph}^{+}\right)$B-ALL patients and patients with
Ferrata Storti Foundation

Haematologica 2019

Volume 104(4):749-755

\section{Correspondence:}

JOSEPH D. KHOURY

jkhoury@mdanderson.org

Received: August 23, 2018.

Accepted: October 22, 2018.

Pre-published: October 25, 2018.

doi:10.3324/haematol.2018.205252

Check the online version for the most updated information on this article, online supplements, and information on authorship \& disclosures: www.haematologica.org/content/104/4/749

\section{(C)2019 Ferrata Storti Foundation}

Material published in Haematologica is covered by copyright. All rights are reserved to the Ferrata Storti Foundation. Use of published material is allowed under the following terms and conditions:

https://creativecommons.org/licenses/by-nc/4.0/legalcode. Copies of published material are allowed for personal or internal use. Sharing published material for non-commercial purposes is subject to the following conditions:

https://creativecommons. org//icenses/by-nc/4.0/legalcode, sect. 3. Reproducing and sharing published material for commercial purposes is not allowed without permission in writing from the publisher. 
$\mathrm{Ph}$-like molecular and cytogenetic signatures are currently treated on frontline protocols with tyrosine kinase inhibitors, which have dramatically improved the outcome of this previously poor prognostic group. ${ }^{1-4}$ Outcomes of patients with T-ALL are generally inferior to those of their B-ALL counterparts, particularly in adults, and the molecular heterogeneity of T-ALL has only recently been uncovered using high-throughput molecular methods. Early T-cell precursor ALL (ETPALL) is a subset of T-ALL that was identified recently and found to include a sizeable proportion of patients with poor outcomes. ${ }^{5,6}$ In contrast to children, only 30$40 \%$ of adults with ALL achieve long-term remission, and survival drops substantially in patients over 60 years of age. ${ }^{7.8}$ Despite advances in frontline treatment of adult ALL, the prognosis of patients who fail frontline and first salvage therapy is extremely poor,10 and justifies the need to explore new therapeutic modalities.

CD123, the interleukin-3 (IL-3) receptor $\alpha$-chain, is the primary low-affinity subunit of the IL-3 receptor and promotes high-affinity binding to IL-3 when co-expressed with the $\beta$-subunit. IL-3 is mainly produced by T-lymphocytes; it regulates the production of hematopoietic cells by stimulating cell cycle progression, differentiation, and inhibition of apoptosis. Early studies suggested that IL-3 plays a critical role in leukemogenesis through enabling leukemic cells to escape programmed cell death and grow autonomously.1 ${ }^{11}$ CD123 was previously reported to be expressed at a low level or to be absent on normal hematopoietic stem cells, but it is expressed at various levels in hematologic malignancies, including hairy cell leukemia, ${ }^{12}$ acute myeloid leukemia, ${ }^{13}$ blastic plasmacytoid dendritic cell neoplasm, ${ }^{14-16}$ and systemic mastocytosis. ${ }^{17}$ Differential overexpression of CD123 by neoplastic cells and their normal precursors has positioned this cell surface receptor as an attractive target of therapy.

The potential of CD123-targeted therapies in ALL remains largely unexplored. There are some data on CD123 expression in B-ALL, but only limited data for TALL. ${ }^{13,15,18}$ In this report, we present a comprehensive single-institution survey of CD123 expression in adult ALL and assess the correlation between CD123 expression and clinicopathological factors and outcomes. We also describe the in vitro impact of IMGN632, a conjugate of CD123-targeting antibody with a novel DNA-alkylating payload, in ALL cell lines and patients' samples.

\section{Methods}

\section{Study group}

A total of 213 consecutive patients (183 with B-ALL, 30 with T-ALL) were identified and included in the study group. B-ALL patients were further subdivided into $\mathrm{Ph}^{+}(121 / 124$ treatmentnaïve) and $\mathrm{Ph}$-negative $\left(\mathrm{Ph}^{-}\right)$(51/59 treatment-naïve) subsets based on cytogenetic, fluorescence in situ hybridization, and/or molecular detection of $\mathrm{t}(9 ; 22)$ (q34.1; $\mathrm{q} 11.2) / B C R-A B L 1$. In the $\mathrm{T}-$ ALL group, 19 patients were treatment-naïve and 11 presented with relapsed/refractory disease after prior treatment. T-ALL patients were subdivided into immunophenotypic subsets based on the expression of CD1a and $\mathrm{SCD} 3$ : the subsets were early $\left(\mathrm{CD}^{-} \mathrm{a}^{-}, \mathrm{sCD}^{-}\right)$, thymic $\left(\mathrm{CD} 1 \mathrm{a}^{+}, \mathrm{sCD} 3^{-}\right)$, or mature $\left(\mathrm{CD} 1 \mathrm{a}^{-}\right.$, $\left.\mathrm{sCD}^{+}\right)$T-ALL. Patients with ETP-ALL were defined as described previously. ${ }^{6}$ Additional details regarding the study group are provided in the Online Supplement. This study was approved by the Institutional Review Board of The University of Texas MD Anderson Cancer Center (MDACC) and conducted in accordance with the Declaration of Helsinki.

\section{Antibodies and reagents}

For the flow cytometry analysis (details below), we used an allophycocyanin-conjugated anti-CD123 (IL-3 receptor $\alpha$ chain) antibody (clone 7G3; BD Pharmingen, BD Biosciences) according to the manufacturer's recommendation. The humanized anti-CD123 antibody G4723A and a humanized non-targeting control antibody of the same IgG1 isotype and identical Fc sequences, were generated at ImmunoGen. IMGN632 and the control DGN549 antibody drug conjugate were produced via conjugation of DGN549 to the G4723A and the non-targeting IgG1 antibodies, respectively, as described previously. ${ }^{19}$

\section{Multicolor/multiparameter flow cytometry analysis}

The blast gate was defined on the basis of CD $45^{\mathrm{dim}}$ expression and side-scatter characteristics and quantified as a percentage of total gated events. For analysis of CD123 expression, measurements included mean fluorescence intensity (MFI) on leukemic blasts (adjusted for background fluorescence using negative internal controls) and relative mean fluorescence intensity (RFI) ratio (leukemic blasts versus non-leukemic events). In patients' samples, positive CD123 expression $\left(\mathrm{CD} 123^{+}\right)$was defined as expression in $\geq 20 \%$ of leukemic blasts using MFI by comparison to background fluorescence and fluorescence on nonleukemic gated events, respectively. Additional details are provided in the Online Supplement.

\section{Cytogenetics and molecular diagnostics}

Conventional cytogenetics, fluorescence in situ hybridization, polymerase chain reaction-based molecular diagnostics, and next-generation sequencing-based mutation profiling were performed on bone marrow aspirate specimens as described previously. ${ }^{20-22}$

\section{Cell lines}

B-ALL cell lines CRF-SB and JM-1 (from American Type Culture Collection) and KOPN-8, SEM, 380, TOM-1, and SD-1 (from Deutsche Sammlung von Mikroorganismen und Zellkulturen) were procured between 2000 and 2015; they were characterized by the respective vendors using DNA profiling. Cytotoxicity was assessed using either a water-soluble tetrazolium salt 8 (WST-8)-based cell viability assay (Dojindo Molecular Technologies) as described previously ${ }^{23}$ or the alamarBlue Cell Viability Reagent (Invitrogen). Further details are given in the Online Supplement.

\section{In vitro evaluation of primary B-cell acute lymphoblas- tic leukemia samples}

Bone marrow mononuclear cells from 11 newly diagnosed and 10 relapsed/refractory B-ALL patients were obtained from MDACC or ConversantBio. The number of CD123 antibodybinding sites per cell ( $A B C$ ) was quantified by the $B D$ QuantiBRITE ${ }^{\mathrm{TM}}$ Fluorescence Quantitation Kit (BD Biosciences) using G4723A conjugated to R-phycoerythrin at a 1:1 ratio, as already described.$^{24}$ Cell proliferation for samples treated with IMGN632 was assessed using the CellTiter-Glo ${ }^{\circledR}$ (Promega) or Cell Trace $^{\mathrm{TM}}$ Violet stain (Invitrogen) techniques. Additional details are provided in the Online Supplement.

\section{Statistical analysis}

The statistical analysis methodology is described in the Online Supplement. 


\section{Results}

\section{Patients' characteristics}

The study group included a total of 213 patients, 131 men and 82 women, with a median age of 40.5 years (range, 1.3-88.3 years) at diagnosis; $25(11.7 \%)$ patients were under 18 years of age (22 with B-ALL and 3 with TALL). For the purposes of this study, patients were divided into three clinically relevant groups: $B C R$ - $A B L 1$-positive $\left(\mathrm{Ph}^{+}\right) \mathrm{B}$-ALL, $B C R$ - $A B L 1$-negative $\left(\mathrm{Ph}^{-}\right) \mathrm{B}$-ALL, and $\mathrm{T}$ ALL. Most $\mathrm{Ph}^{+}$patients $(42 / 59 ; 71.2 \%)$ expressed the e1a2 BCR-ABL1 fusion transcript, followed by the b2a2 and b3a2 types. Detailed comparisons of the clinical and laboratory features of the $\mathrm{CD}_{123^{+}}$and $\mathrm{CD} 123^{-}$patients in the three groups are provided in Table 1.
CD123 expression by multicolor/multiparameter flow cytometry in patients' samples

The median number of blasts detected by multicolor/multiparameter flow cytometry across the entire study group was $76.5 \%$ (range, 7.2-98.0\%) and did not differ between the three groups $(P=0.872)$. When the entire group was considered, the median percentage of CD $45^{\text {dim }}$ blasts expressing CD123 was $64.7 \%$ (range, $0.3-$ $99.9 \%$ ), and the median RFI was 11.0 (range, $0.0-420.5$ ). CD123 positivity ( $>20 \%$ blasts) was seen in 177 (83\%) of all ALL patients. CD123 ${ }^{+}$blasts were positive for CD34 in 58/59 (98.3\%) $\mathrm{Ph}^{+} \mathrm{B}-\mathrm{ALL}$, in 95/124 (76.6\%) $\mathrm{Ph}^{-} \mathrm{B}$-ALL and in $13 / 30(43.3 \%)$ T-ALL patients. We identified a strong correlation between $\mathrm{CD} 123$ and $\mathrm{CD} 34$ expression on leukemic blasts across the entire study group $(\mathrm{r}=0.483 ; P<0.0001)$.

Table 1. Clinical and laboratory features of patients in the study group $(n=213)$ categorized by CD123 expression status.

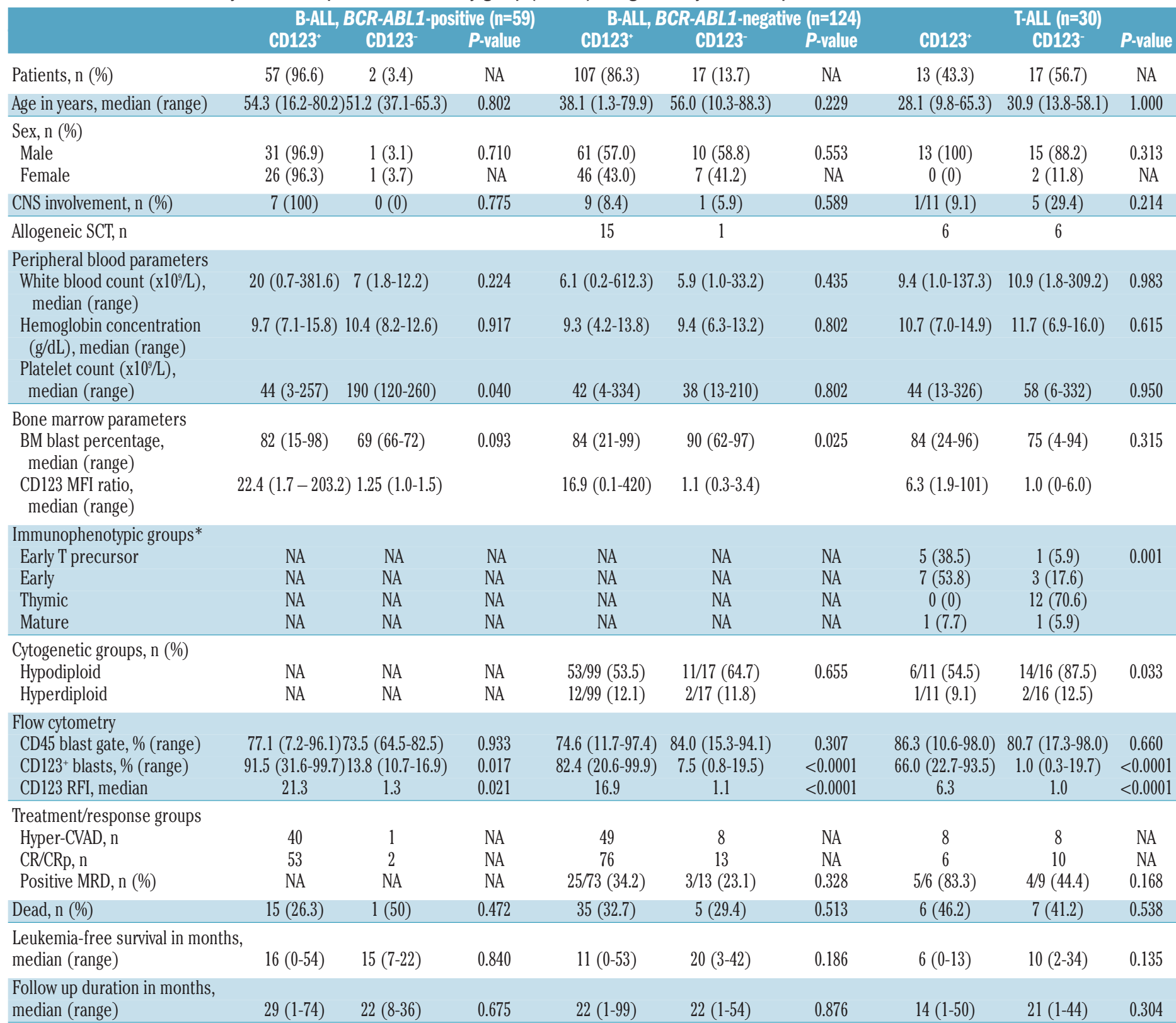

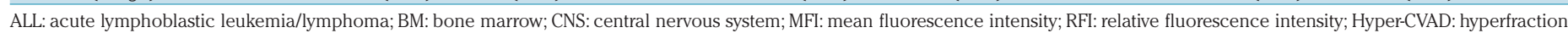

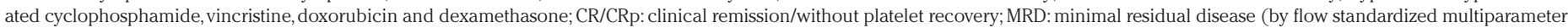
flow cytometry analysis). NA: not-applicable, SCT: stem cell transplantation; $P$-values refer to comparisons of CD123+ and CD123- patients within a given clinical subgroup. 
The frequency of CD123 expression was higher in BALL than in T-ALL (164/183, 89.6\% versus 13/30, 43.3\%; $P<0.0001)$, and within B-ALL the frequency of CD123 expression was higher in $\mathrm{Ph}^{+}$versus $\mathrm{Ph}^{-} \mathrm{B}$-ALL patients (96.6\% versus $86.3 \% ; P=0.033$ ). (Figure $1 \mathrm{~A}$ ) The intensity of CD123 expression across groups of patients showed similar patterns. Namely, the median CD123 RFI was higher in B-ALL compared to T-ALL (14.45 versus 2.35; $P<0.001)$, and within B-ALL it tended to be higher in $\mathrm{Ph}^{+}$ compared with $\mathrm{Ph}^{-}$patients, although the difference did not attain statistical significance (20.85 versus 11.8; $P=0.08$ ) (Figure $1 \mathrm{~B}$ ). We analyzed our findings using various CD123 MFI-based cutoffs of $20 \%$, 30\% 50\%, as well as RFI $\geq 10$. All conclusions remained valid at cutoff levels of $20 \%$ and $30 \%$ as well as RFI $\geq 10$ but not at the $50 \%$ cutoff level (data not shown).

In the T-ALL group (40\% thymic; 33\% early non-ETP; $20 \%$ ETP; $7 \%$ mature), the median CD123+ blast proportion was $17.95 \%$ (range, $0.5-93 \%$ ) with a median RFI of 2.35 (range, $0-101)$. We identified a correlation $(P=0.001)$ between T-ALL immunophenotypic subgroups and CD123 expression status; namely, 12/13 (92.3\%) patients with $\mathrm{CD}_{123^{+}}$T-ALL had either ETP or early non-ETP immunophenotype.

\section{Correlation between CD123 expression and clinical parameters}

CD123 expression status did not correlate with minimal residual disease status at the end of induction. The median follow-up duration for the entire group was 23 months (range, 1-79 months) and was comparable for patients with $\mathrm{CD}_{123^{+}}$and $\mathrm{CD} 123^{-}$ALL. There was no difference in Performance Status between $\mathrm{CD}_{123^{+}}$and CD123- ALL patients in any of the clinical subgroups (data not shown). At last follow up, 69 (32.4\%) patients were dead. There was no difference in overall survival between patients with $\mathrm{CD}_{123^{+}}$and $\mathrm{CD} 123^{-}$ALL within any of the subgroups. However, for T-ALL patients, the relapse-free survival rate was more favorable in those with CD123- disease compared with those with $\mathrm{CD}^{-123^{+}}$ disease $(P=0.03)$ (Online Supplementary Figure S1). Similar results were obtained when patients under 18 years of age were excluded. Notably, the impact of CD123 expression on relapse-free survival correlated with the intensity of CD123 expression in adult T-ALL using RFI $\geq 10$ vs. $<10$ as a cutoff $(P=0.015)$ (Online Supplementary Figure S2). For patients with B-ALL, CD123 expression showed no correlation with relapse-free survival (Online Supplementary Figure S3).

We performed multivariate analysis using Cox regression modeling (Wald backward stepwise method) to evaluate the impact of CD123 expression status on relapse-free survival alongside other prognostic variables that included: minimal residual disease status at complete remission, central nervous system involvement, and age (patients $<18$ years excluded). Analyses were conducted in each of the clinical subgroups separately. In the $\mathrm{Ph}^{+} \mathrm{B}$-ALL group only minimal residual disease status at complete remission was independently associated with relapse-free survival $(P=0.019)$, and in the $\mathrm{Ph}^{-} \mathrm{B}$-ALL group only central nervous system involvement was independently associated with relapse-free survival $(P=0.005)$. In the T-ALL group, none of the variables was associated with leukemia-free survival.

\section{In vitro anti-leukemia activity of IMGN632 in acute lymphoblastic leukemia}

IMGN632 is a conjugate of the CD123-binding antibody G4723A with a novel DNA-alkylating IGN payload, DGN549. The numbers of binding sites for the antibody component of IMGN632 were quantified on leukemic blasts and on lymphocytes from 21 patients with newly diagnosed or relapsed/refractory B-ALL (Online Supplementary Figure S4). As described above, the blast population was identified based on the CD $45^{\mathrm{dim}} / \mathrm{SSC}^{\text {low/med}} / \mathrm{CD} 19^{+} / \mathrm{CD} 34^{+}$profile, while the lymphocyte population was identified based on CD $45^{\text {bright }} / S_{S C}{ }^{\text {low }}$ characteristics. Of the 21 samples analyzed, 20 expressed the CD123 antigen, with a median number of $\mathrm{ABC}$ of 1,085 on leukemic blasts and 57 on lymphocytes. There was no appreciable difference in the number of $A B C$ between blasts from newly diagnosed and relapsed/ refractory patients.

The cytotoxicity of IMGN632 was assessed in a panel of
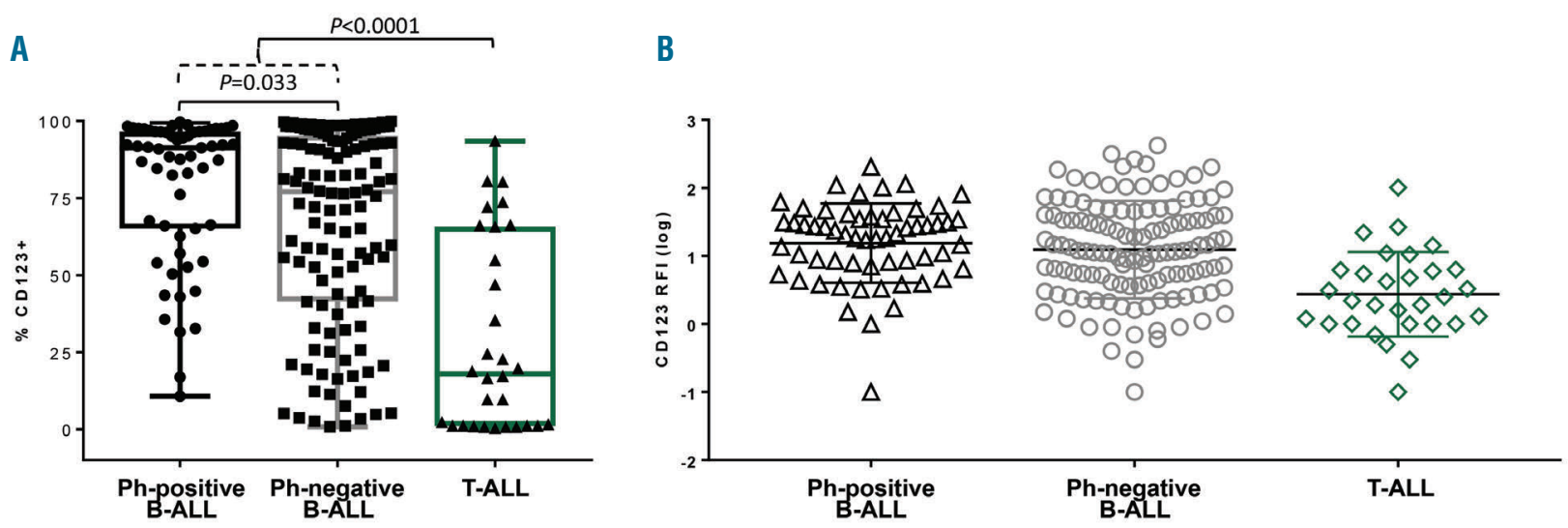

Figure 1. Expression of CD123 on leukemic blasts in B and T acute lymphoblastic leukemia/lymphoma by multiparameter flow cytometry immunophenotyping. (A) Percentage of leukemic blasts with CD123 expression based on mean fluorescence intensity relative to background in each of the groups of patients. CD123 expression was significantly different between B-acute lymphoblastic leukemia/lymphoma (ALL) and T-ALL ( $P<0.0001$; Mann-Whitney U test). In addition, CD123 expression was significantly different between Philadelphia chromosome ( $\mathrm{Ph}$ )-positive and Ph-negative patients ( $P=0.033$; Mann-Whitney $U$ test). (Lines: median, $25^{\text {th }}-75^{\text {th }}$ percentiles). (B) Relative fluorescence intensity (RFI) on leukemic blasts compared to non-leukemic gated events (lines: mean \pm standard deviation). Ph: Philadelphia chromosome and/or BCR/ABL1 fusion status. 
B-ALL cell lines that expressed CD123 at levels similar to those of blasts from B-ALL patients (ABC values: 457$3,741)$ and encompassed genetic variants associated with a poor prognosis (Table 2). IMGN632 was highly cytotoxic to B-ALL cell lines with half maximal inhibitory concentrations $\left(\mathrm{IC}_{50}\right.$ ) between 0.6 and $20 \mathrm{pM}$. The conjugate cytotoxicity was CD123-specific, as dosing with a non-targeting conjugate (Ab-DGN549) prepared with a similar linker and payload reduced the potency more than 1000-fold.

IMGN632 potency was evaluated in primary samples from eight patients with B-ALL. The blast cells in each of the untreated samples proliferated in culture, as manifested by a dramatic decrease in the cell trace violet fluorescent signal, while only a small shift in fluorescence (likely due to nonspecific dissociation of the dye) was observed in the lymphocyte population, suggesting lack of proliferation (Figure 2A). IMGN632 eliminated more than $90 \%$ of the BALL blast population in five of eight samples, including those from newly diagnosed as well as relapsed/refractory patients, at low pico-molar concentrations (Figures 2B,C). Normal lymphocytes were not affected by IMGN632 at 100-fold higher concentrations.

\section{Discussion}

In this study, we demonstrate pervasive CD123 expression in a large cohort of ALL patients. In B-ALL, previously reported to be often $\mathrm{CD} 123^{+}, 13,15,18$ our findings demonstrate that CD123 expression translates into susceptibility to targeting CD123 via the antibody-drug conjugate IMGN632 in vitro and ex vivo. Coupled with previously published data on the differential overexpression of CD123 on the surface of acute myeloid leukemia blasts, but not on normal hematopoietic stem cells, ${ }^{25}$ these findings provide a plausible basis for the exploration of anti-CD123 therapy in B-ALL patients in whom frontline treatment fails. Furthermore, in T-ALL our findings suggest that CD123 expression might be associated with an early precursor immunophenotype, including ETP-ALL. Since the latter is a high-risk subset, targeting CD123 in these patients might be of potential benefit if the association is confirmed in a larger group of ETP-ALL patients.

Previously, the CD123-targeting conjugate IMGN632 was reported to have high potency in pre-clinical models of acute myeloid leukemia. ${ }^{24}$ In this study we demonstrated the efficacy of IMGN632 in pre-clinical B-ALL models, suggesting that IMGN632 is a promising anti- leukemia agent in CD123-expressing ALL. In addition to its promising anti-leukemia activity, CD123 offers an alternative to CD19-targeting therapeutic approaches to circumvent CD19 antigen-loss relapses and/or elimination of CD19- ${ }^{-}$stem cells. ${ }^{26}$

B-ALL is a heterogeneous disease that includes nine genetic subgroups recognized in the World Health Organization classification. ${ }^{27}$ Data on correlations between CD123 expression and B-ALL subgroups are limited. Djokic et al. showed that B-ALL cases with a hyperdiploid karyotype have significantly higher rates of CD123 expression compared to other subgroups such as BCR/ABL1, MLL, ETV6/RUNX1 and diploid karyotype. ${ }^{18}$ However, their study included mostly pediatric patients, and the $B C R / A B L 1$ subgroup formed a small proportion of their cohort (2/81 pediatric patients; 3/13 adult patients). In this study, we identified a significant correlation between CD123 expression and the presence of the $B C R / A B L 1$ fusion, with a higher percentage of $\mathrm{CD}_{123^{+}}$events and a higher intensity in comparison to the $\mathrm{Ph}^{-} \mathrm{B}-\mathrm{ALL}$ and T-ALL groups. The association between the BCR/ABL1 fusion and CD123 has also been identified in chronic myeloid leukemia, in which targeted CD123 inhibition appears to deplete chronic myeloid leukemia progenitor and stem cells. ${ }^{28,29}$ The findings in this study suggest a close correlation between $B C R / A B L 1$ and CD123, this time in the context of B-ALL. Whether the correlation between $\mathrm{Ph}^{-} \mathrm{B}-\mathrm{ALL}$ and $\mathrm{CD} 123$ is related to an underlying Ph-like biology remains to be determined and is being pursued in an ongoing separate study.

There are limited and somewhat controversial data on CD123 expression in T-ALL. Several prior studies have suggested that CD123 is not or only scarcely expressed in T-ALL, although most of these studies had relatively small sample sizes. ${ }^{13,15,18}$ Two other studies reported expression of CD123 by T-ALL blasts. ${ }^{30,31}$ In a study by Lhermitte et al. CD123 expression was detected in 16\% of T-ALL; notably, CD123 positivity was reported to be less frequent in children than in adults ( $9 \%$ versus $23 \%) .{ }^{31}$ Another study of a large cohort of T-ALL patients by Du et al. found a slightly higher frequency of CD123 expression and noted an age-dependent difference, further suggesting a lower frequency of CD123 expression in pediatric patients $(27 \%$ versus $42 \%) .^{30}$ A crucial difference precluding direct comparison of the results of those studies with the current study is lack of data on CD123 intensity (RFI) in the earlier studies.

ETP-ALL comprises a unique subset of T-ALL previously belonging to the subset of early T-ALL. ETP ALL

Table 2. In vitro cytotoxicity of IMGN632 in B-acute lymphoblastic leukemia/lymphoma cell lines.

\begin{tabular}{|c|c|c|c|}
\hline Cell line & Genetic variant & $\begin{array}{l}\text { TMGN632 } \\
\left(\mathrm{IC}_{50} \mathrm{pM}\right)\end{array}$ & $\begin{array}{c}\text { Ab-DGN549 } \\
\left(\mathrm{IC}_{50} \mathrm{pM}\right)\end{array}$ \\
\hline CCRF-SB & EBNA-positive & 20 & 20,000 \\
\hline JM1 & & 10 & 20,000 \\
\hline KOPN-8 & $\mathrm{t}(11 ; 19)(\mathrm{q} 23 ; \mathrm{p} 13) / K M T 2 A-M L L T 1$ & 6 & 13,000 \\
\hline SEM & $\mathrm{t}(4 ; 11) / K M T 2 A-A F F 1$ & 3 & 20,000 \\
\hline 380 & $\mathrm{t}(8 ; 14) / M Y C-I G H$ and $\mathrm{t}(14 ; 18) / I G H-B C L 2$ EBNA-negative & 2 & 7,000 \\
\hline TOM-1 & $\mathrm{t}(9 ; 22)(\mathrm{q} 34.1 ; \mathrm{q} 11.2) / B C R-A B L 1$ fusion gene (e1-a2) & 2 & 20,000 \\
\hline SD-1 & $\mathrm{t}(9 ; 22)(\mathrm{q} 34.1 ; \mathrm{q} 11.2) / B C R-A B L 1$ fusion gene (e1-a2) & 0.6 & 3,000 \\
\hline
\end{tabular}

$\mathrm{IC}_{50}$ : half maximal inhibitory concentration; pM: pico-males. 
has been reported in 10-13\% of childhood T-ALL and in $5-17 \%$ of adults. ${ }^{5,6}$ Jain et al. reported significantly poorer outcomes in patients with ETP-ALL, with lower complete remission and 5 -year overall survival rates, compared with other T-ALL patients. ${ }^{6}$ In the present study, patients with ETP-ALL represented $20 \%$ of the T-ALL cohort. We postulate that this high rate of ETP-ALL is likely due, in part, to higher detection rates and to a referral bias to our institution in view of the higher relapse rates of patients with this subtype. Only limited data, using different stratification approaches, are available from other studies on the correlation between CD123 expression and immunophenotype in T-ALL. Lhermitte et al. stratified patients with T-ALL on the basis of a cytoplasmic T-cell receptor $\beta$-negative phenotype and patterns of TRD, TRG and TRB configurations, ${ }^{31}$ as opposed to commonly used multicolor/multiparame- ter flow cytometry immunophenotyping criteria according to the World Health Organization classification. These authors found that CD123 expression was more common in "immature" T-ALL, being detectable in $68 \%$ of adults and $36 \%$ of children; in contrast, they identified CD123 expression in 5\% of "non-immature" T-ALL cases. Du et al. classified T-ALL patients into "early $\mathrm{T}$ precursor" $\left(\mathrm{CD}^{+}\right)$, "T-precursor" $\left(\mathrm{CD} 2^{+}\right.$and/or $\mathrm{CD}^{+}$ and/or $\left.\mathrm{CD}^{+}\right)$, and "mature" $\left(\mathrm{CD}^{+}\right),{ }^{30}$ according to the European Group for the Immunological Characterization of Leukemias (EGIL) criteria. In their study, 83\% of cases with an "early T-precursor" immunophenotype were positive for CD123. In keeping with those previous observations, our ETP-ALL group - as well as early-nonETP - showed the highest frequency of CD123 expression compared with the thymic and mature immunophenotypes.
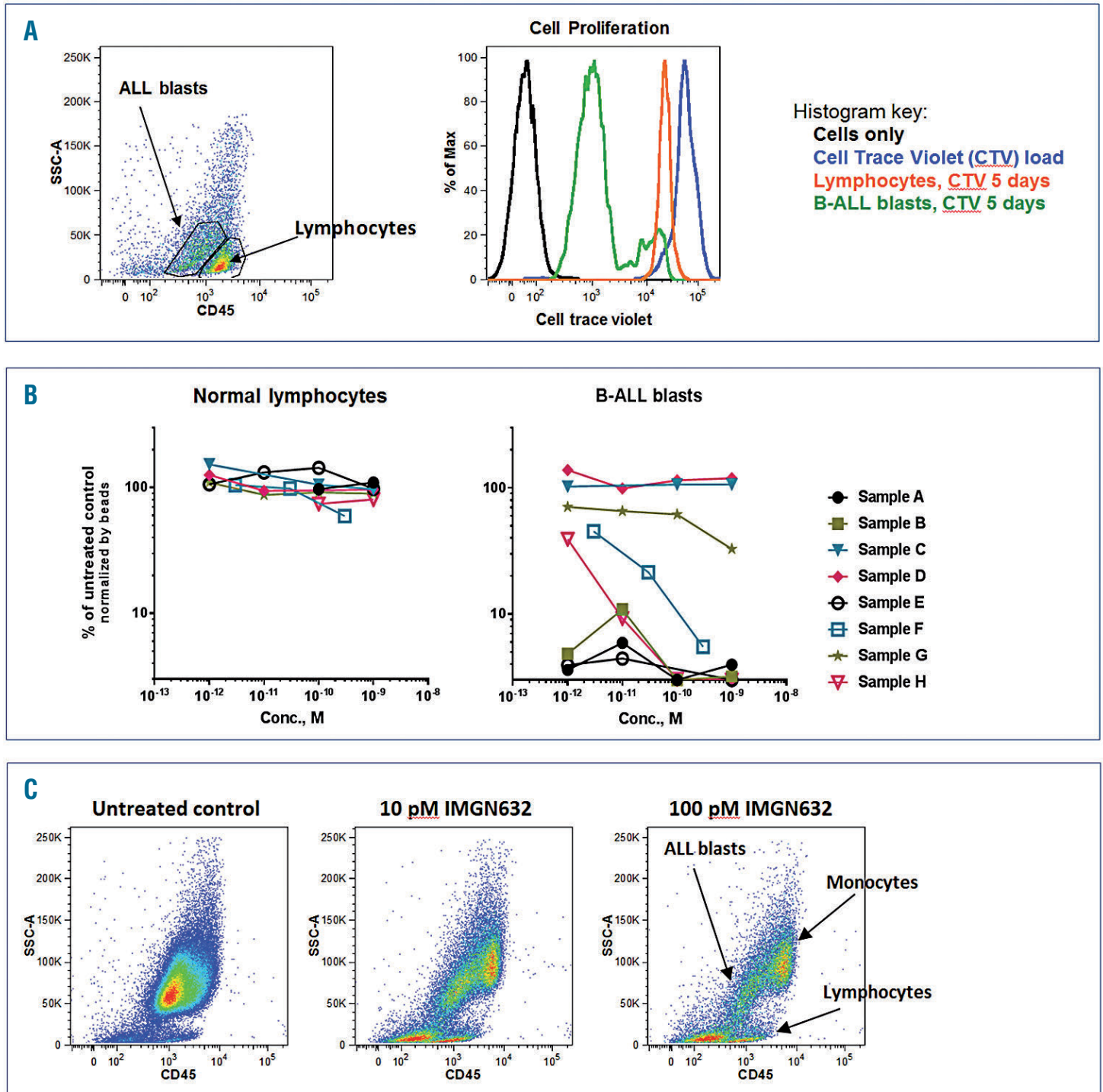

Figure 2. In vitro potency of IMGN632 on leukemic blasts cells and lymphocytes from patients with B-acute lymphoblastic leukemia/lymphoma. (A) The leukemic blast population, but not lymphocyte population, proliferates in culture. (B) IMGN632 is cytotoxic to leukemic blasts from five of eight patients with B-acute lymphoblastic leukemia/lymphoma (B-ALL), while sparing lymphocytes. (C) IMGN632 eliminates blasts, but not lymphocytes in a specimen (sample H) from a patient with relapsed-refractory B-ALL. 
Although a higher number of $\mathrm{CD}_{123^{+}}$leukemic stem cells was shown to have a negative impact on leukemiafree and overall survival in patients with acute myeloid leukemia, ${ }^{25,32}$ information on the prognostic impact of CD123 expression in ALL has been limited and unclear. In a large study of pediatric ALL, high-level CD123 expression was most common in patients with a hyperdiploid karyotype, a favorable prognostic marker. ${ }^{18}$ We did not identify such a correlation in this study group of mostly adult patients. In T-ALL patients, CD123 expression was not found to be prognostic of outcome after first induction treatment. ${ }^{30}$ In our study group, we did not identify a cor- relation between CD123 prevalence and leukemia-free survival or overall survival in B-ALL, but we did observe a significant correlation between the intensity of CD123 expression and leukemia-free survival. We acknowledge that this finding in a limited group of patients warrants further validation in a larger cohort.

In conclusion, data from this study show that CD123 is widely expressed in B-ALL and to a lesser degree in TALL, and they confirm its potential utility as a therapeutic target. IMGN632, an antibody-drug conjugate which targets CD123, has promising preclinical activity against B-ALL.

\section{References}

1. Roberts KG, Gu Z, Payne-Turner D, et al. High frequency and poor outcome of Philadelphia chromosome-like acute lymphoblastic leukemia in adults. J Clin Oncol. 2017;35(4):394-401.

2. Jain N, Roberts KG, Jabbour E, et al. Ph-like acute lymphoblastic leukemia: a high-risk subtype in adults. Blood. 2017;129(5):572581.

3. Roberts KG, Li Y, Payne-Turner D, et al. Targetable kinase-activating lesions in $\mathrm{Ph}$ like acute lymphoblastic leukemia. N Engl J Med. 2014;371(11):1005-1015.

4. El Fakih R, Jabbour E, Ravandi F, et al. Current paradigms in the management of Philadelphia chromosome positive acute lymphoblastic leukemia in adults. Am J Hematol. 2018;93(2):286-295.

5. Coustan-Smith E, Mullighan CG, Onciu M, et al. Early T-cell precursor leukaemia: a subtype of very high-risk acute lymphoblastic leukaemia. Lancet Oncol. 2009;10(2):147 156.

6. Jain N, Lamb AV, O'Brien S, et al. Early T-cell precursor acute lymphoblastic leukemia/lymphoma (ETP-ALL/LBL) in adolescents and adults: a high-risk subtype. Blood. 2016;127(15):1863-1869.

7. Sive JI, Buck G, Fielding A, et al. Outcomes in older adults with acute lymphoblastic leukaemia (ALL): results from the international MRC UKALL XII/ECOG2993 trial. Br J Haematol. 2012;157(4):463-471.

8. Jabbour E, O'Brien S, Konopleva M, Kantariian $\mathrm{H}$. New insights into the pathophysiology and therapy of adult acute lymphoblastic leukemia. Cancer. 2015;121(15): 2517-2528.

9. O'Brien S, Thomas D, Ravandi F, et al. Outcome of adults with acute lymphocytic leukemia after second salvage therapy. Cancer. 2008;113(11):3186-3191.

10. Gokbuget N, Stanze D, Beck J, et al Outcome of relapsed adult lymphoblastic leukemia depends on response to salvage chemotherapy, prognostic factors, and performance of stem cell transplantation. Blood. 2012;120(10):2032-2041.

11. Lotem J, Cragoe EJ, Jr., Sachs L. Rescue from programmed cell death in leukemic and normal myeloid cells. Blood. 1991;78(4):953960.
12. Del Giudice I, Matutes E, Morilla R, et al. The diagnostic value of CD123 in B-cell disorders with hairy or villous lymphocytes. Haematologica. 2004;89(3):303-308.

13. Munoz L, Nomdedeu JF, Lopez $O$, et al. Interleukin-3 receptor alpha chain (CD123) is widely expressed in hematologic malignancies. Haematologica. 2001;86(12):12611269.

14. Angelot-Delettre F, Roggy A, Frankel AE, et al. In vivo and in vitro sensitivity of blastic plasmacytoid dendritic cell neoplasm to SL401 , an interleukin-3 receptor targeted biologic agent. Haematologica. 2015;100(2): 223-230.

15. Testa U, Pelosi E, Frankel A. CD 123 is a membrane biomarker and a therapeutic target in hematologic malignancies. Biomark Res. 2014;2(1):4

16. Alayed K, Patel KP, Konoplev S, et al. TET2 mutations, myelodysplastic features, and a distinct immunoprofile characterize blastic plasmacytoid dendritic cell neoplasm in the bone marrow. Am J Hematol. 2013;88(12): 1055-1061.

17. Pardanani A, Reichard KK, Zblewski D, et al. CD123 immunostaining patterns in systemic mastocytosis: differential expression in disease subgroups and potential prognostic value. Leukemia. 2016;30(4):914-918.

18. Djokic M, Bjorklund E, Blennow E, Mazur J, Soderhall S, Porwit A. Overexpression of CD123 correlates with the hyperdiploid genotype in acute lymphoblastic leukemia. Haematologica. 2009;94(7):1016-1019.

19. Miller ML, Fishkin NE, Li W, et al. A new class of antibody-drug conjugates with potent DNA alkylating activity. Mol Cancer Ther. 2016;15(8):1870-1878.

20. Zhang L, Singh RR, Patel KP, et al. BRAF kinase domain mutations are present in a subset of chronic myelomonocytic leukemia with wild-type RAS. Am J Hematol. 2014;89(5):499-504.

21. Khoury JD, Sen F, Abruzzo LV, Hayes K, Glassman A, Medeiros LJ. Cytogenetic findings in blastoid mantle cell lymphoma. Hum Pathol. 2003;34(10):1022-1029.

22. Salem A, Loghavi S, Tang G, et al. Myeloid neoplasms with concurrent BCR-ABL1 and CBFB rearrangements: a series of 10 cases of a clinically aggressive neoplasm. Am J Hematol. 2017;92(6):520-528.

23. Kovtun YV, Audette CA, Mayo MF, et al.
Antibody-maytansinoid conjugates designed to bypass multidrug resistance. Cancer Res. 2010;70(6):2528-2537.

24. Kovtun Y, Jones GE, Adams S, et al. A CD123-targeting antibody-drug conjugate, IMGN632, designed to eradicate AML while sparing normal bone marrow cells. Blood Adv. 2018;2(8):848-858.

25. Testa U, Riccioni R, Militi S, et al. Elevated expression of IL-3Ralpha in acute myelogenous leukemia is associated with enhanced blast proliferation, increased cellularity, and poor prognosis. Blood. 2002;100(8):29802988.

26. Ruella M, Barrett DM, Kenderian SS, et al. Dual CD19 and CD123 targeting prevents antigen-loss relapses after CD19-directed immunotherapies. J Clin Invest. 2016;126 (10):3814-3826

27. Borowitz MJ, Chan JKC, Downing JR, Le Beau MM, Arber DA, Bene MC. Precursor lymphoid neoplasms. . In: Swerdlow SH, Campo E, Harris NL, et al., eds. WHO Classification of Tumours of Haematopoietic and Lymphoid Tissues Lyon: IARC, 2017:200-213.

28. Nievergall E, Ramshaw HS, Yong AS, et al. Monoclonal antibody targeting of IL-3 receptor alpha with CSL362 effectively depletes CML progenitor and stem cells. Blood. 2014;123(8):1218-1228.

29. Frolova O, Benito J, Brooks C, et al. SL-401 and SL-501, targeted therapeutics directed at the interleukin-3 receptor, inhibit the growth of leukaemic cells and stem cells in advanced phase chronic myeloid leukaemia. Br J Haematol. 2014;166(6):862-874.

30. Du W, Li J, Liu W, et al. Interleukin-3 receptor alpha chain (CD123) is preferentially expressed in immature T-ALL and may not associate with outcomes of chemotherapy. Tumour Biol. 2016;37(3):3817-3821.

31. Lhermitte L, de Labarthe A, Dupret C, et al. Most immature T-ALLs express Ra-IL3 (CD123): possible target for DT-IL3 therapy. Leukemia. 2006;20(10):1908-1910.

32. Vergez F, Green AS, Tamburini J, et al. High levels of CD34+CD38low/-CD123+ blasts are predictive of an adverse outcome in acute myeloid leukemia: a Groupe OuestEst des Leucemies Aigues et Maladies du Sang (GOELAMS) study. Haematologica. 2011;96(12):1792-1798. 